\title{
Evaluation of Digital Mammography in Diagnosis of Breast Cancer
}

\author{
Sigeru Nawano
}

\begin{abstract}
Mammography has been digitized in all cases at our hospital. Digital mammography (MMG) of our hospital and its diagnostic accuracy were described in this report. Fuji Computed Radiography (FCR; Fuji Medical Systems, Tokyo, Japan) imaging plate was used and imaging data were processed with FCR 7000 or FCR 9000. Each image was output to a single hard copy. Sampling pitches for reading and output were $0.1 \mathrm{~mm}$. The rate of breast cancer diagnosis by digital MMG was $67 \%, 95 \%, 94 \%$, and $100 \%$ for unpalpable tumor, tumor less than $\phi 2 \mathrm{~cm}$, tumor of $\phi 2$ to $5 \mathrm{~cm}$, and tumor greater than $\phi 5 \mathrm{~cm}$, respectively, being $94 \%$ overall. Digital MMG enables us to establish goal-oriented image-processing conditions. The use of digital MMG, which provides an excellent diagnostic rate similar to that of screen-film MMG, is expected to became widespread in the near future.

Copyright $\odot 1995$ by W.B. Saunders Company
\end{abstract}

KEY WORDS: digital mammography, breast cancer, computer-aided diagnosis.

$\mathbf{T}$ HE RECENT remarkable advances in digital imaging have allowed our hospital (National Cancer Center Hospital East, Chiba, Japan) to digitize $90 \%$ of its radiographs since opening. Mammography (MMG) has been digitized in all cases; currently, film-screen mammography is not being prescribed at all. The present report describes digital MMG and its diagnostic accuracy.

\section{MAMMOGRAPHIC TECHNIQUE}

Digital MMG was performed using MGU-01 (Toshiba, Tokyo, Japan) and MAMMOMAT 3 (Siemens Medical Systems, Inc, Iselin, NJ) mammographs with grids. In craniocaudal and mediolateral oblique viewing directions, an $8-\times 10$-in Fuji Computed Radiography (FCR; Fuji Medical Systems, Tokyo, Japan) imaging plate was used and imaging data were processed with FCR 7000 or FCR 9000. Each image was output to a single hard copy. Sampling pitches for reading and output were $0.1 \mathrm{~mm}$. Our own in-house image processing conditions were used. For craniocaudal and mediolateral views, adjustments were made to make the contrast and concentration curve equivalent to those of Ortho $M$ film and Min-R screen system (Eastman Kodak, Rochester, NY) (Fig 1). Since January

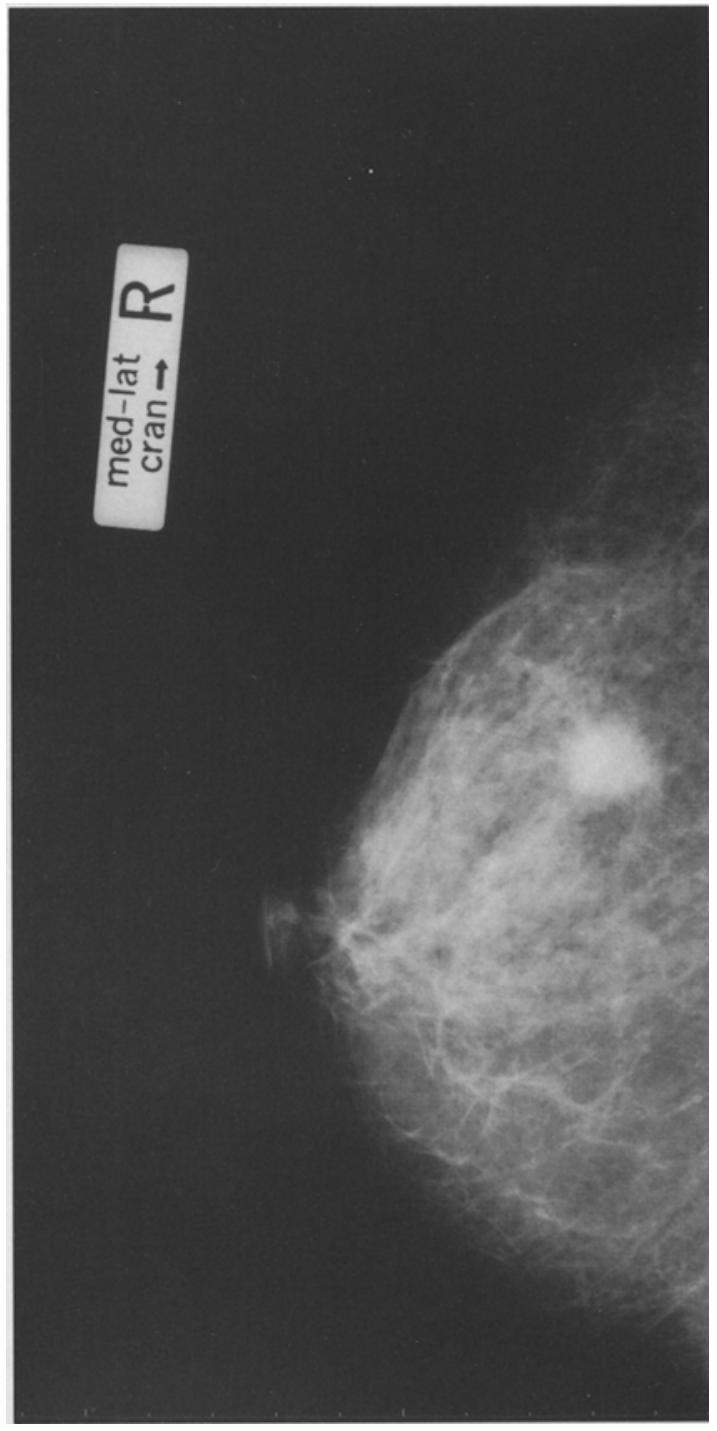

Fig 1. Right mediolateral view shows a high-density spiculated mass in the upper outer quadrant. Results of operation showed infiltrating ductal carcinoma.

From the Department of Radiology, National Cancer Center Hospital, East Chiba, Japan.

Address reprint requests to Sigeru Nawano, MD, Department of Radiology, National Cancer Center Hospital East, 6-5-1, Kashiwanoha, Kashiwa City, Chiba 277, Japan.

Copyright $\odot 1995$ by W.B. Saunders Company 0897-1889/95/0801-1015\$3.00/0 
1994, digital MMG combined with dynamic range-compression processing (DCP) has led to significant improvement in the resolution of skin margin while maintaining clear contrast in the focal region ${ }^{1,2}$ (Fig 2). Spot viewing is occasionally added if necessary, and in this case, 1.7-fold enlargement viewing was performed without using grid. Image-processing conditions in spot viewing were set up with consideration of granularity, and the concentration curve was set so as to be equivalent to Min-R film, Min-R screen (Fig 3). Images were evaluated by an experienced radiologist at the time of digital
MMG examination, and, if necessary, the spot was also palpated before preparing the report.

\section{BREAST CANCER DIAGNOSTIC RATE (SENSITIVITY) OF DIGITAL MMG}

The rate of breast cancer diagnosis by digital MMG was $67 \%, 95 \%, 94 \%$, and $100 \%$ for unpalpable tumor, tumor $<\phi 2 \mathrm{~cm}$, tumor of $\phi$ 2 to $5 \mathrm{~cm}$, and tumor $>\phi 5 \mathrm{~cm}$, respectively, being $94 \%$ overall in cases correctly diagnosed as positive for malignancy and possible malignancy. Tumor-related calcification among these was observed in $40 \%$ of the patients (intratumor
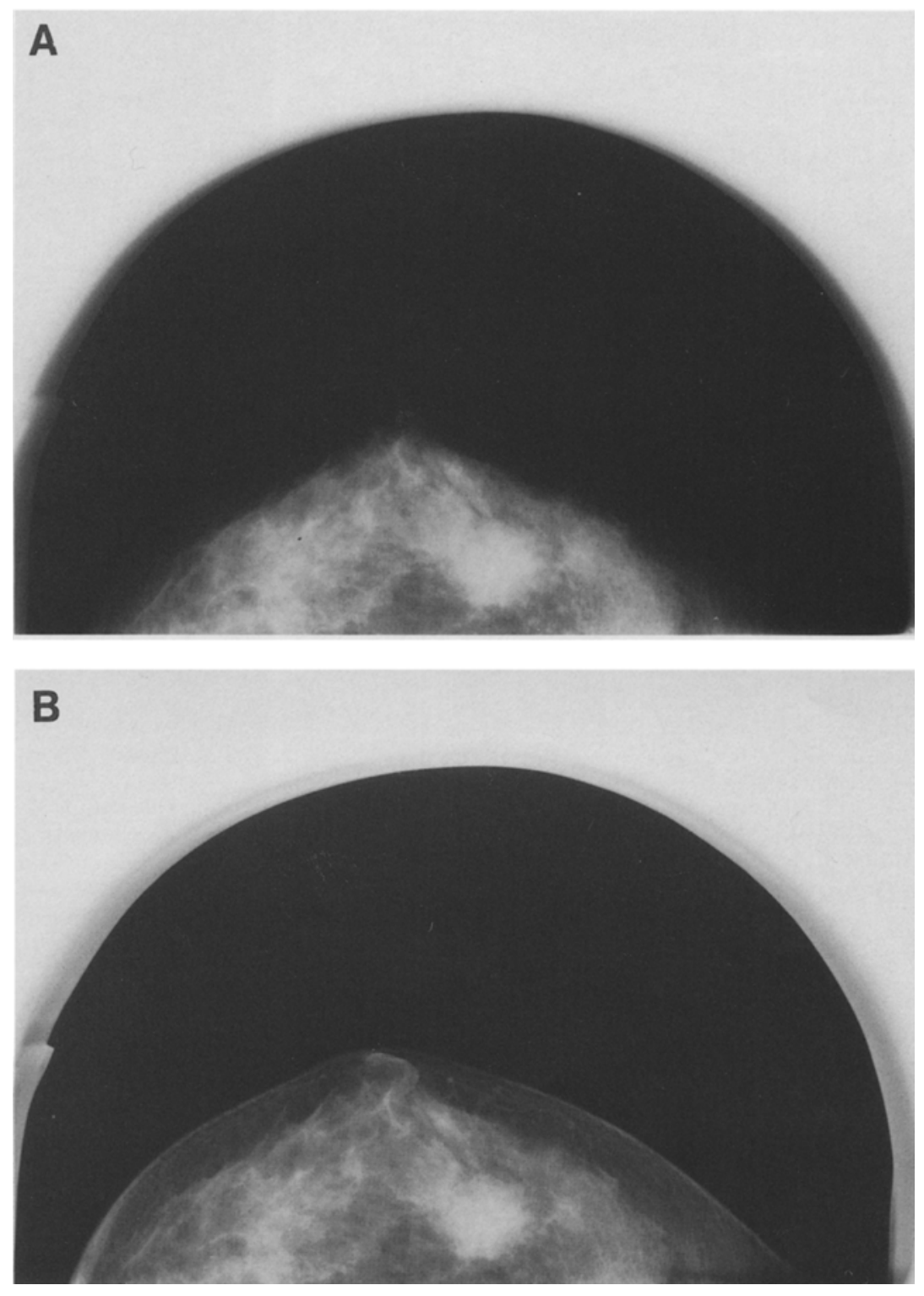

Fig 2. (A) There is a highdensity irregular mass (breast cancer) in the outer aspect on left craniocaudal view. (B) Dynamic range compression processing image showing not only breast cancer but also subcutaneal tissue. 
Fig 3. Spot viewing shows high-density mass with fine microcalcifications and spicules characteristic of malignancy.

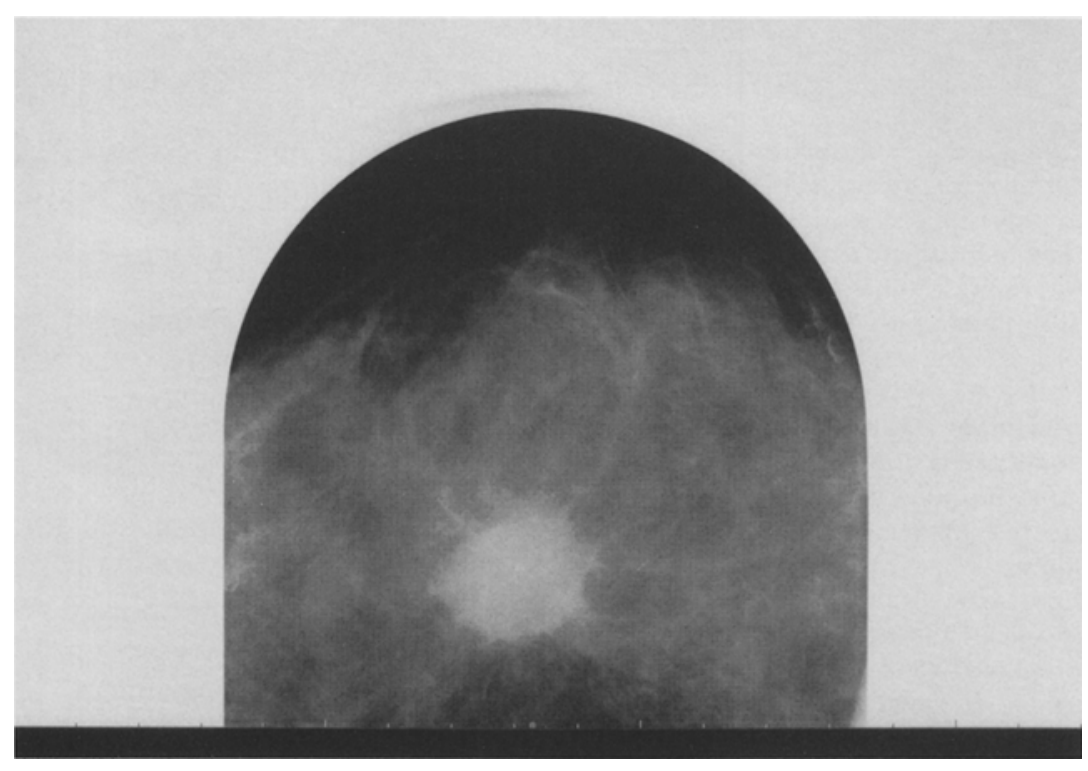

calcification, 35\%; nontumor forming, diffuse calcification, $5 \%$ ).

\section{DIAGNOSTIC RATE OF DIGITAL-MMG WITHIN A DEFINITE PERIOD}

Twenty-six cases of breast cancer were detected among 149 cases undergoing digital MMG during a 3-month period beginning in September 1992. A sensitivity of $92 \%$ and specificity of $91 \%$ were obtained with an accuracy of $91 \%$.

\section{COMPUTER-AIDED DIAGNOSIS OF BREAST CANCER BY MMG PROCESSING}

A joint study performed with Kobatake enabled us to develop a computerized system for breast cancer diagnosis. ${ }^{3}$ The tumor detection rate in typical cases was represented by a true-positive rate of $100 \%$ and false-positive rate of $24 \%$. For the diagnosis of micro calcification, a true-positive rate of $100 \%$ was obtained in typical clustered cases.

\section{CONCLUSION}

Digital MMG enables us not only to establish goal-oriented image-processing conditions, but also to add DCP filter processing, which provides good visualization of both high- and lowdensity regions on the mammogram. The use of digital MMG, which provides an excellent diagnostic rate similar to that of screen-film mammography, is expected to became widespread in the near future.

\section{REFERENCES}

1. Yoshyhiro Anan, Takashi Ootani, Shigeru Nawano, et al: Development of dynamic-range conversion processing. Image Technol Inform Display 23:805-811, 1991

2. Yoshyhiro Anan, Takashi Ootani, Shigeru Nawano, et al: Application of dynamic-range conversion processing to mammography. Image Technol Inform Display 24:663-668, 1992
3. Hidehumi Kobatake, Hua-Ron Jin, Yukiharu Yoshinaga, et al: Computer diagnosis of breast cancer by mammogram processing. Proceeding of the International Symposium on Computer-Assisted Radiology. Berlin, Germany, Springer-Verlag, 1993, pp 624-629 